\title{
Fractional iteration in the disk algebra: prime ends and composition operators
}

\section{Manuel D. Contreras and Santiago Díaz-Madrigal}

\begin{abstract}
In this paper we characterize the semigroups of analytic functions in the unit disk which lead to semigroups of operators in the disk algebra. These characterizations involve analytic as well as geometric aspects of the iterates and they are strongly related to the classical theorem of Carathéodory about local connection and boundary behaviour of univalent functions.
\end{abstract}

\section{Introduction and statement of the results}

Let $\mathcal{H}(\mathbb{D})$ be the Fréchet space of all analytic functions in the disk endowed with the topology of uniform convergence on compact subsets of $\mathbb{D}$. A (oneparameter) semigroup of $\mathcal{H}(\mathbb{D})$ or, a semigroup of analytic functions, is any continuous homomorphism $\Phi: t \mapsto \Phi(t)=\varphi_{t}$ from the additive semigroup of nonnegative real numbers $\mathbb{R}^{+}$into the composition semigroup of all functions $\varphi \in \mathcal{H}(\mathbb{D})$ with $\varphi(\mathbb{D}) \subset \mathbb{D}$. That is, $\Phi$ satisfies the following three conditions:

a) $\varphi_{0}$ is the identity in $\mathbb{D}$,

b) $\varphi_{t+s}=\varphi_{t} \circ \varphi_{s}$, for all $t, s \geq 0$,

c) For every $s \geq 0, \varphi_{t}$ tends to $\varphi_{s}$ in the topology of $\mathcal{H}(\mathbb{D})$, as $t$ tends to $s$.

It is well-known that condition c) can be replaced by

c') For every $z \in \mathbb{D}, \lim _{t \rightarrow 0} \varphi_{t}(z)=z$.

Semigroups of $\mathcal{H}(\mathbb{D})$ are deeply related to the theory of composition operators. Namely, given a Banach space $X$ formed by functions of $\mathcal{H}(\mathbb{D})$

2000 Mathematics Subject Classification: Primary: 30C45, 30D40, 30H05, 47B33, 47D06. Secondary: 54D05.

Keywords: Semigroups, disk algebra, prime end, starlike functions, spirallike functions. 
and a semigroup of analytic functions $\Phi=\left(\varphi_{t}\right)$, it is said that $\Phi$ generates a semigroup of operators in $X$ if the family $\left(T_{t}\right)$ of composition operators

$$
f \in X \longrightarrow T_{t}(f):=f \circ \varphi_{t} \in \mathcal{H}(\mathbb{D}) \quad(t \geq 0),
$$

is a strongly continuous semigroup of bounded operators in $X[5$, Chapter VIII]. This opens the possibility of studying spectral properties, operator ideal properties or dynamical properties of the semigroup of operators $\left(T_{t}\right)$ in terms of the theory of functions. The paper [1] of Berkson and Porta can be considered the starting point of this branch of Analysis.

Clearly, a first problem appears: which semigroups of analytic functions generate semigroups of operators in $X$ ? Classical choices of $X$ treated in the literature [13] are the Hardy spaces $H_{p}(\mathbb{D})(1 \leq p \leq \infty)$, the disk algebra $A(\mathbb{D})$, the Bergman spaces $A_{p}(\mathbb{D})(1 \leq p \leq \infty)$, the Dirichlet space $\mathcal{D}$, the Bloch space, the space $B M O A(\mathbb{D})$, and the space $V M O A(\mathbb{D})$. In some cases, the answer to the above question is known (see [11] and references therein). In fact,

(i) Every semigroup of $\mathcal{H}(\mathbb{D})$ generates a semigroup of operators in $H_{p}(\mathbb{D})$ $(1 \leq p<\infty), A_{p}(\mathbb{D})(1 \leq p<\infty), \mathcal{D}$, and $\operatorname{VMOA}(\mathbb{D})$.

(ii) None non-trivial semigroup of $\mathcal{H}(\mathbb{D})$ generates a semigroup of operators in $H_{\infty}(\mathbb{D})$ and the Bloch space. We point out that a semigroup $\Phi=$ $\left(\varphi_{t}\right)$ of $\mathcal{H}(\mathbb{D})$ is said to be trivial if each $\varphi_{t}$ is the identity in $\mathbb{D}$.

(iii) The above question for $B M O A(\mathbb{D})$ seems to be open. It is known that there exist semigroups of $\mathcal{H}(\mathbb{D})$ that do not generate semigroups of operators in that Banach space. But, it is even unknown if there is a non-trivial semigroup in $\mathcal{H}(\mathbb{D})$ leading to a semigroup of operators in $B M O A(\mathbb{D})$.

As far as we know, the situation for the disk algebra is even worse than in (iii). What is more, Siskakis [11] conjectured that, for this case, we are in a situation similar to $($ iii). However, there is no even a correct example of a semigroup of $\mathcal{H}(\mathbb{D})$ which does not lead to a semigroup of operators in $A(\mathbb{D})$.

As usual, the disk algebra $A(\mathbb{D})$ is the Banach space of all analytic functions in $\mathbb{D}$ with continuous extensions to $\overline{\mathbb{D}}$ endowed with the supremum norm.

The main goal of this paper is to characterize the semigroups of analytic functions which generate semigroups of operators in the disk algebra and, as a by-product, to provide a number of examples of semigroups of $\mathcal{H}(\mathbb{D})$ which generate semigroups of operators in every $H_{p}(\mathbb{D})(1 \leq p<\infty)$ but not in $A(\mathbb{D})$. These characterizations involve analytic as well as geometric aspects of the functions $\left(\varphi_{t}\right)$ and they are strongly related to a classical theorem of Carathéodory. 
Our first theorem is based on standard results and arguments.

Theorem 1.1 Let $\Phi=\left(\varphi_{t}\right)$ be a semigroup in $\mathcal{H}(\mathbb{D})$. The following assertions are equivalent.

1. $\Phi$ generates a semigroup of operators in the disk algebra.

2. $\varphi_{t} \in A(\mathbb{D})$, for all $t \geq 0$, and $\lim _{t \rightarrow s}\left\|\varphi_{t}-\varphi_{s}\right\|_{\infty}=0$, for all $s \geq 0$.

3. $\varphi_{t} \in A(\mathbb{D})$, for all $t \geq 0$, and $\lim _{t \rightarrow 0} \varphi_{t}(z)=z$, for every $z \in \overline{\mathbb{D}}$.

4. $\varphi_{t} \in A(\mathbb{D})$, for all $t \geq 0$, and $\lim _{t \rightarrow 0} \varphi_{t}(z)=z$, for every $z \in \partial \mathbb{D}$.

The equivalence between (1) and (2) is given in [11, p. 236]. It is worth comparing statements (3) and (4) with condition c') in the definition of semigroups of analytic functions. In some sense, this theorem is a first step to a deeper criterion for the so-called semigroups of $\mathcal{H}(\mathbb{D})$ with inner DWpoint (inner Denjoy-Wolff point) or with boundary DW-point (boundary Denjoy-Wolff point). These concepts require some dynamical background and, for a detailed exposition, we refer to [11].

Anyway, it is a fundamental fact that every non-trivial semigroup of analytic functions $\Phi=\left(\varphi_{t}\right)$ is of one of the following mutually exclusive types:

- Neutral DW-point: The family of analytic functions $\left(\varphi_{t}\right)$ has a unique common fixed point $b \in \mathbb{D}$ such that $\left|\varphi_{t}^{\prime}(b)\right|=1$, for all $t \geq 0$.

- Inner DW-point: The family of analytic functions $\left(\varphi_{t}\right)_{t>0}$ has a unique common fixed point $b \in \mathbb{D}$ which is attractive.

- Boundary $D W$-point: The family of analytic functions $\left(\varphi_{t}\right)_{t>0}$ has a unique common fixed point $b \in \partial \mathbb{D}$ which is attractive.

It is known that semigroups with neutral DW-point are formed by elliptic automorphisms of the disk or the identity. So, bearing in mind Theorem 1.1 and $[10$, p. 108], it is immediate that this type of semigroups always generates a semigroup of operators in $A(\mathbb{D})$. Therefore, the problem we are dealing with is of real interest only for the other two types.

Semigroups $\left(\varphi_{t}\right)$ with inner DW-point can be described perfectly by means of the theory of univalent functions: assuming that $b \in \mathbb{D}$ is the corresponding common fixed point, there is a unique univalent function $h: \mathbb{D} \rightarrow \mathbb{C}$ with $h(0)=0$ and $h^{\prime}(0)=1$, verifying the geometric condition

$$
\begin{gathered}
\text { "there is } c \in \mathbb{C} \text { with } \operatorname{Re}(c)>0 \text { such that, for each } t>0, \\
e^{-c t} \Omega \subset \Omega \text { where } \Omega:=h(\mathbb{D}) "
\end{gathered}
$$

and such that

$$
\varphi_{t}(z)=h_{b}^{-1}\left(e^{-c t} h_{b}(z)\right), \quad t \geq 0, z \in \mathbb{D},
$$

where $h_{b}=h \circ m_{b}$. 
As usual, $m_{b}$ is the elliptic automorphism of the disk associated to $b$, that is,

$$
m_{b}(z)=\frac{b-z}{1-\bar{b} z}, \quad z \in \mathbb{D} .
$$

The set $\Omega=h(\mathbb{D}) \subset \mathbb{C}$ is called the associated planar domain of $\Phi$.

It is worth mentioning that semigroups with neutral DW-point can be described as above but with $\operatorname{Re} c=0$ and $c \neq 0$. In this case, the corresponding planar domain $\Omega$ is $\lambda \mathbb{D}$ for some $\lambda \geq 0$.

A similar description can be given for semigroups $\left(\varphi_{t}\right)$ with boundary DW-point: assuming that $b \in \partial \mathbb{D}$ is the corresponding common fixed point, there is a unique univalent function $h: \mathbb{D} \rightarrow \mathbb{C}$ with $h(0)=0$ verifying the geometric condition

$$
\text { "for each } t>0, \Omega+t \subset \Omega \text { where } \Omega:=h(\mathbb{D}) \text { " }
$$

and such that

$$
\varphi_{t}(z)=b h^{-1}(h(\bar{b} z)+t), \quad t \geq 0, z \in \mathbb{D} .
$$

Again, the set $\Omega=h(\mathbb{D}) \subset \mathbb{C}$ is called the associated planar domain of $\Phi$.

For our next theorem, we need the topological ingredient of local connection. We recall that a topological space is said to be locally connected if every point of the space has a neighborhood basis formed by open and connected subsets. This concept admits some nice equivalences in the context of compact metric spaces (see [3, p. 48]). Namely, a compact metric space $(Y, d)$ is locally connected if for every $\varepsilon>0$ there is $\delta>0$ such that whenever $x$ and $y$ are points in $Y$ with $d(x, y)<\delta$, there is a connected subset $A$ of $Y$ containing $x$ and $y$ and satisfying $\operatorname{diam} A<\varepsilon$. The key which relates these topological and functional ideas is the continuity theorem of Carathéodory: An univalent function $h$ of $\mathcal{H}(\mathbb{D})$ can be extended continuously to $\overline{\mathbb{D}}$ if and only if the boundary of $h(\mathbb{D})$ (in the Riemann sphere $\mathbb{C}_{\infty}$ ) is locally connected.

In what follows, $\partial_{\infty} \Omega$ denotes the boundary of the set $\Omega$ in $\mathbb{C}_{\infty}$. Now we come to our main result.

Theorem 1.2 Let $\Phi=\left(\varphi_{t}\right)$ be a semigroup of analytic functions and let $\Omega$ be the corresponding planar domain. The following assertions are equivalent.

1. $\Phi$ generates a semigroup of operators in the disk algebra.

2. $\partial_{\infty} \Omega$ is locally connected.

3. Every $\varphi_{t}$ belongs to the disk algebra. 
Now, we can present the desired example. Consider a sequence $\left(\theta_{n}\right)_{n \geq 1} \backslash 0$, $\theta_{0}=0$, and the bounded starlike (with respect to zero) domain

$$
\Omega=\mathbb{D} \backslash\left(\bigcup_{n \geq 0}[0.4,1] e^{i \theta_{n}}\right)
$$

whose boundary is not locally connected (see Figure 1).

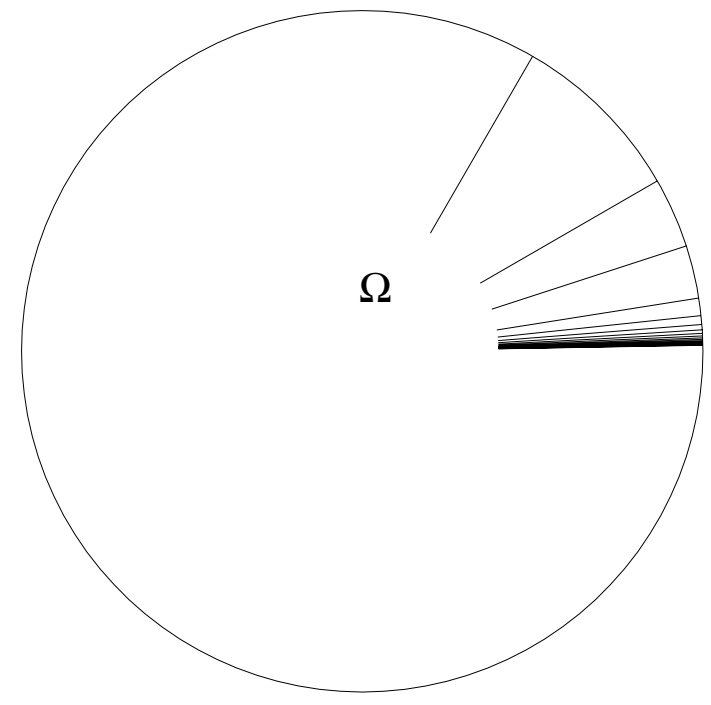

FiguRE 1: Starlike domain whose boundary is not locally connected.

Take the Riemann map $h$ from $\mathbb{D}$ onto $\Omega$ with $h(0)=0$ and $h^{\prime}(0)=1$ and define

$$
\varphi_{t}(z):=h^{-1}\left(e^{-t} h(z)\right), \quad t \geq 0, z \in \mathbb{D} .
$$

Then, using Theorem 1.2, we see that $\left(\varphi_{t}\right)$ is a semigroup of analytic functions which generates a semigroup of operators in every $H_{p}(\mathbb{D})(1 \leq p<\infty)$ but not in $A(\mathbb{D})$.

Remark 1.1. It is well-known that $\partial_{\infty} \Omega$ is locally connected if and only if there is a curve in $\mathbb{C}_{\infty}$ such that its range is exactly $\partial_{\infty} \Omega$ (see [3], [7]).

Remark 1.2. In general, it is not true that the statement (3) can be replaced by "There exists $t>0$ such that $\varphi_{t} \in A(\mathbb{D})$ ". For instance, if $\Omega$ is bounded (even with $\partial_{\infty} \Omega$ non locally connected) and $b=0$ is the corresponding DW-point of $\Phi$, it is clear that we can always find $t>0$ such that $e^{-t} \bar{\Omega} \subset \Omega$, so trivially, $\varphi_{t} \in A(\mathbb{D})$. Anyway, looking at the implication $(2) \Rightarrow(3)$ in the proof of Theorem 1.2, it is easy to find examples of unbounded domains $\Omega$ whose boundary $\partial_{\infty} \Omega$ is not locally connected and such that $\varphi_{t} \notin A(\mathbb{D})$ for all $t>0$ and, of course, the semigroup $\left(\varphi_{t}\right)$ generates a semigroup of operators in $H_{p}(\mathbb{D})(1 \leq p<\infty)$. 
If we combine our theorems with the continuity theorem of Carathéodory and with [7, Theorem 9.11] (see also [8, pp. 22-23]), we obtain the following.

Corollary 1.3 Let $\left(\varphi_{t}\right)$ be a semigroup of analytic functions with inner $D W$-point. Then the following are equivalent.

1. Every $\varphi_{t} \in A(\mathbb{D})$ and $\varphi_{t} \rightarrow \varphi_{t_{0}}$ uniformly on $\overline{\mathbb{D}}$, for all $t_{0} \geq 0$.

2. For each $T>0$, the family $\left(\mathbb{C} \backslash \varphi_{t}(\mathbb{D})\right)_{t \in[0, T]}$ of subsets of $\mathbb{C}$ is uniformly locally connected. That is, for every $\varepsilon>0$ there is $\delta>0$ such that whenever $0 \leq t \leq T$ and $x$ and $y$ are points in $\mathbb{C} \backslash \varphi_{t}(\mathbb{D})$ with $|x-y|<\delta$, there is a connected subset $A$ of $\mathbb{C} \backslash \varphi_{t}(\mathbb{D})$ containing $x$ and $y$ and satisfying $\operatorname{diam} A<\varepsilon$.

3. Every $\varphi_{t} \in A(\mathbb{D})$.

4. Every $\mathbb{C} \backslash \varphi_{t}(\mathbb{D})$ is locally connected.

According to well-known results of Carathéodory the equivalences (1) $\Leftrightarrow$ (2) and $(3) \Leftrightarrow(4)$ separately looks foreseeable. What is more surprising is that the four assertions are equivalent. Of course, this global equivalence depends strongly on deep results of the theory of semigroups of operators in Banach spaces.

Remarks about the proof of Theorem 1.2. To prove Theorem 1.2 we have to distinguish the three cases: neutral DW-point, inner DW-point, and boundary DW-point. Of course, after Theorem 1.1, the theorem is trivial for the neutral DW-case. As far as we know, it is not possible to obtain the proof of one of the other two cases as a consequence of the other (by means of some kind of reduction) due to the fact that they have essentially different multipliers from the dynamic point of view. So, we should present a proof for the inner DW-point and a proof for the boundary DW-point. However, we are going to present only the inner DW-case. The reason is that both proofs are basically the same, changing arguments with logarithmic spirals for arguments with half-lines. The level of similarity is so high that to write both proofs yields to duplicate arguments in a large number of pages.

So, for the sake of clarity, we present only a very detailed proof of the DW-inner case.

Acknowledgments. The authors thank D. Vukotic for a number of fruitful discussions on the content of this paper. The authors also want to thank the referee for his/her comments and specially for suggesting Proposition 4.1 which simplifies and improves the exposition of the first version of this paper. Our arguments of the first version where based completely on prime end theory. 


\section{Proof of Theorem 1.1}

Proof. (of Theorem 1.1) It is clear that $(1) \Rightarrow(2) \Rightarrow(3) \Rightarrow(4)$. We just have to prove that $(4) \Rightarrow(1)$.

First of all, since $\varphi_{t} \in A(\mathbb{D})$ for all $t \geq 0$, we know that each composition operator $T_{t}$ is continuous in the disk algebra. Now, bearing in mind the characterization of the strong continuity of a semigroup in terms of the weak operator topology (see, for example, [12, p. 233]), it is enough to prove that, for every $f \in A(\mathbb{D})$, it is true that

$$
w-\lim _{t \searrow 0} T_{t}(f)=f,
$$

where $w$ denotes the weak topology of $A(\mathbb{D})$.

So, let us fix a sequence of positive real numbers $t_{n}$ which goes to zero. We must show that given $f \in A(\mathbb{D})$, the sequence $\left(f \circ \varphi_{t_{n}}\right)$ converges to $f$ in the weak topology of $A(\mathbb{D})$. In order to prove this, let us recall that, in the space of continuous functions on the boundary of the unit disk, $C(\partial \mathbb{D})$, given a bounded sequence $\left(g_{n}\right)$ and $g \in C(\partial \mathbb{D})$, we have that $\left(g_{n}\right)$ converges to $g$ in the weak topology of $C(\partial \mathbb{D})$ if and only if $g_{n}(z) \rightarrow g(z)$ for all $z \in \partial \mathbb{D}$ (see, for example, [4, p. 66]). Now, since the disk algebra is a closed subspace of $C(\partial \mathbb{D})$, we have the same characterization for the convergence of bounded sequences in the weak topology of $A(\mathbb{D})$. In our case, we take the sequence $g_{n}=f \circ \varphi_{t_{n}}$ which is bounded since $\left\|f \circ \varphi_{t_{n}}\right\|_{\infty} \leq\|f\|_{\infty}$.

Hence we have to get that $f \circ \varphi_{t_{n}}(z) \rightarrow f(z)$ for all $z \in \partial \mathbb{D}$ and for all $f \in A(\mathbb{D})$. By (4), this is true for the identity function $f(z)=z$. Now, if $m \geq 0$, we have that

$$
\left|\varphi_{t_{n}}(z)^{m}-z^{m}\right| \leq m\left|\varphi_{t_{n}}(z)-z\right| \underset{n \rightarrow \infty}{\rightarrow} 0 \quad \text { for all } z \in \partial \mathbb{D} .
$$

From this, it is clear to get that given an analytic polynomial $p$, we have that

$$
\left|p\left(\varphi_{t_{n}}(z)\right)-p(z)\right| \underset{n \rightarrow \infty}{\rightarrow} 0
$$

for all $z \in \partial \mathbb{D}$. Finally, when $f$ is an arbitrary function of $A(\mathbb{D}), z \in \partial \mathbb{D}$, and $\varepsilon>0$, we can find an analytic polynomial $p$ such that $\|f-p\|_{\infty}<\varepsilon / 3$. For this polynomial there is a natural number $n_{0}$ such that if $n \geq n_{0}$ we have that $\left|p\left(\varphi_{t_{n}}(z)\right)-p(z)\right|<\varepsilon / 3$. Then

$$
\begin{aligned}
\left|f\left(\varphi_{t_{n}}(z)\right)-f(z)\right| \leq\left|f\left(\varphi_{t_{n}}(z)\right)-p\left(\varphi_{t_{n}}(z)\right)\right| & \\
& \quad+\left|p\left(\varphi_{t_{n}}(z)\right)-p(z)\right|+|f(z)-p(z)| \\
& \leq\|f-p\|_{\infty}+\left|p\left(\varphi_{t_{n}}(z)\right)-p(z)\right|+\|f-p\|_{\infty}<\varepsilon .
\end{aligned}
$$

That is, $f \circ \varphi_{t_{n}}(z) \rightarrow f(z)$ for all $z \in \partial \mathbb{D}$. 


\section{Spirallike domains and prime ends}

Let $h$ be a univalent function of the unit disk $\mathbb{D}$ onto a simply connected domain $\Omega \subset \mathbb{C}$. In what follows, we shall see $\Omega$ as a subset of the Riemann sphere $\mathbb{C}_{\infty}$ and use the spherical metric $d^{\#}$ in $\mathbb{C}_{\infty}$. Carathéodory introduced the concept of a prime end of $\Omega$ in order to describe the boundary behaviour of $h$ in geometric terms. We are going to present some well-known facts and notations about prime ends in order the paper to be self-contained and more readable. The reader can see a detailed exposition and proofs of these facts in the books by Conway [3], Milnor [6], and Pommerenke [7], [8]. Whenever $\Omega$ contains 0 , a crosscut of $\Omega$ is a Jordan arc $C$ in $\Omega$ such that the end points of $\bar{C}^{\mathbb{C}_{\infty}}$ lie on $\partial_{\infty} \Omega$. In this case, $\Omega \backslash C$ has two connected components and the portion of the boundary of each of these components that lies in $\Omega$ is $C$. If a crosscut does not pass through 0 , let out $C$ denote the component of $\Omega \backslash C$ that contains 0 and let ins $C$ denote the other component. From now on we will only consider crosscuts of $\Omega$ that do not pass through 0 . A zero-chain of $\Omega$ is a sequence of crosscuts of $\Omega,\left(C_{n}\right)$, having the following properties:

a: $\operatorname{ins} C_{n+1} \subset \operatorname{ins} C_{n}$;

b: ${\overline{C_{n}}}^{\mathbb{C}_{\infty}} \cap{\overline{C_{m}}}^{\mathbb{C}_{\infty}}=\emptyset$ for $n \neq m ;$

c: $\operatorname{diam}^{\#} C_{n} \rightarrow 0$ as $n \rightarrow \infty$.

If $\left(C_{n}\right)$ and $\left(C_{n}^{\prime}\right)$ are two zero-chains in $\Omega$ we say that they are equivalent if for every $n$ there is an $m$ such that ins $C_{m} \subset$ ins $C_{n}^{\prime}$ and, conversely, for every $i$ there is a $j$ with ins $C_{j}^{\prime} \subset \operatorname{ins} C_{i}$. It is easy to see that this concept of equivalence for zero-chains in $\Omega$ is indeed an equivalence relation. A prime end is an equivalence class of zero-chains. Let $\widehat{\Omega}$ denote $\Omega$ together with the collection of prime ends. The Carathéodory topology $T(\widehat{\Omega})$ in $\widehat{\Omega}$ can be described as follows: a subset $U$ of $\widehat{\Omega}$ is open if $U \bigcap \Omega$ is open in $\Omega$ and for every point $p \in U \backslash \Omega$ there exists a zero-chain $\left(C_{n}\right)$ in $p$ such that there is an integer $n$ with ins $C_{n} \subset U \cap \Omega$. It is not difficult to prove that given $\left(w_{m}\right)$ a sequence in $\Omega$ and $p=\left[\left(C_{n}\right)\right]$ a prime end, we have that $w_{m} \rightarrow p$ in $T(\widehat{\Omega})$ if and only if for every $n$ there is an $m_{0}$ such that if $m \geq m_{0}$ it is satisfied that $w_{m} \in \operatorname{ins} C_{n}$.

Now we can state the prime end theorem: $h$ always extends to an homeomorphism $\widehat{h}$ of $\overline{\mathbb{D}}$ onto $\widehat{\Omega}$. Namely, given a prime end $p=\left[\left(C_{n}\right)\right]$ of $\Omega$, it is satisfied that $\left[\left(h^{-1}\left(C_{n}\right)\right)\right]$ is a prime end in $\mathbb{D}$ and there is a unique $z \in \partial \mathbb{D}$ such that $\{z\}=\bigcap_{n} \overline{\operatorname{ins} h^{-1}\left(C_{n}\right)}$ and, in fact, $\widehat{h}(z)=\left[\left(C_{n}\right)\right]$. 
Given $z \in \partial \mathbb{D}$, the impression of the prime end $\widehat{h}(z)=\left[\left(C_{n}\right)\right]$ is the continuum

$$
\begin{array}{r}
I(\widehat{h}(z))=\bigcap_{n \geq 1}{\overline{\operatorname{ins} C_{n}}}^{\mathbb{C}_{\infty}}=\left\{w \in \partial_{\infty} \Omega: \text { there is } z_{n} \in \mathbb{D} \text { with } z_{n} \rightarrow z\right. \\
\text { and } \left.h\left(z_{n}\right) \rightarrow w \text { as } n \rightarrow \infty\right\} .
\end{array}
$$

It is clear that $h$ can be extended to $\mathbb{D} \bigcup\{z\}$ in a continuous way if and only if $I(\widehat{h}(z))$ is singleton.

Whether it is possible to extend $h$ to a continuous function from $\overline{\mathbb{D}}$ into $\mathbb{C}_{\infty}$ has a completely topological answer. Namely, the continuity theorem says that $h$ has a continuous extension to $\overline{\mathbb{D}}$ if and only if $\partial_{\infty} \Omega$ is locally connected. Moreover, an attentive reading of the proof of this theorem shows that $\partial_{\infty} \Omega$ is locally connected if and only if for every prime end $p$ in $\widehat{\Omega}$ and every $\left(C_{n}\right) \in p$, we have that $\operatorname{diam}^{\#}\left(\operatorname{ins} C_{n}\right.$ ) goes to zero.

Notice that, in general, the extension given by the continuity theorem is not an homeomorphism (compare with the prime end theorem). In fact, Carathéodory theorem states that this extension is an homeomorphism if and only if $\partial_{\infty} \Omega$ is a Jordan curve (in $\mathbb{C}_{\infty}$ ).

The following two useful lemmata about the boundary behaviour of the Riemann map of a domain whose boundary is locally connected are probably known. The second one is a slight generalization of $[9, \mathrm{p} .162]$.

Lemma 3.1 Suppose $h$ is a univalent function from $\mathbb{D}$ onto $\Omega$ with $h(0)=0$ and $\partial_{\infty} \Omega$ locally connected. If $w \in \partial_{\infty} \Omega \cap \mathbb{C}$ has exactly one pre-image by $h$, then $h^{-1}: \Omega \rightarrow \mathbb{D}$ has a continuous extension from $\Omega \bigcup\{w\}$ into $\overline{\mathbb{D}}$.

Proof. Since $\partial_{\infty} \Omega$ is a locally connected subset of $\mathbb{C}_{\infty}$, we can assume that $h$ has a continuous extension (which we still denote by $h$ ) from $\overline{\mathbb{D}}$ into $\bar{\Omega} \mathbb{C}_{\infty}$ and $h(\partial \mathbb{D})=\partial_{\infty} \Omega$. Take $z \in \partial \mathbb{D}$ such that $h(z)=w$ and $\left(w_{n}\right)$ in $\Omega$ such that $w_{n} \rightarrow w$. We are going to prove that the sequence $\left(h^{-1}\left(w_{n}\right)\right)$ tends to $z$. By the compactness of $\overline{\mathbb{D}}$, it is enough to get that $\left(h^{-1}\left(w_{n}\right)\right)$ has a subsequence converging to $z$. Take $\left(w_{n_{k}}\right)$ such that $\left(h^{-1}\left(w_{n_{k}}\right)\right)$ converges to a point $b \in \overline{\mathbb{D}}$. Since $h$ is continuous on $\overline{\mathbb{D}}, h(b)=\lim _{k} h\left(h^{-1}\left(w_{n_{k}}\right)\right)=\lim _{k} w_{n_{k}}=w$. Now, $w=h(b)=h(z)$ and, by hypothesis, $b=z$.

Lemma 3.2 Suppose $h$ is a univalent function from $\mathbb{D}$ onto $\Omega$ with $h(0)=0$ and $\partial_{\infty} \Omega$ locally connected. If $\gamma:[0,1) \rightarrow \Omega$ is a curve with $\lim _{s \rightarrow 1^{-}} \gamma(s)=$ $w \in \partial_{\infty} \Omega$, then there exists $z \in \partial \mathbb{D}$ such that

$$
\lim _{s \rightarrow 1^{-}} h^{-1}(\gamma(s))=z
$$


Proof. As in Lemma 3.1, we can view $h$ as a continuous function from $\overline{\mathbb{D}}$ onto $\bar{\Omega}^{\mathbb{C}_{\infty}}$. Consider the $\omega$-limit

$$
\omega\left(h^{-1}(\gamma)\right)=\left\{z \in \overline{\mathbb{D}}: \text { there is } s_{n} \nearrow 1 \text { with } h^{-1}\left(\gamma\left(s_{n}\right)\right) \rightarrow z\right\} .
$$

Since $h$ is continuous in $\mathbb{D}$, we have that $\omega\left(h^{-1}(\gamma)\right)$ is a compact, connected, and non-empty subset of $\partial \mathbb{D}$. Therefore, $\omega\left(h^{-1}(\gamma)\right)$ is a single point or contains a subarc of $\partial \mathbb{D}$ of positive length.

Given $z \in \omega\left(h^{-1}(\gamma)\right)$, we have that, by definition, there is $s_{n} \nearrow 1$ with $h^{-1}\left(\gamma\left(s_{n}\right)\right) \rightarrow z$. So, bearing in mind that $h$ is continuous at $z$,

$$
\gamma\left(s_{n}\right)=h\left(h^{-1}\left(\gamma\left(s_{n}\right)\right)\right) \rightarrow h(z) .
$$

On the other hand, $\gamma\left(s_{n}\right) \rightarrow w$. Therefore, $h(z)=w$ for all $z \in \omega\left(h^{-1}(\gamma)\right)$. Since $h$ can not be a. e. constant on any subarc of $\partial \mathbb{D}$ of positive length $[9$, p. 162], we have that $\omega\left(h^{-1}(\gamma)\right)$ is a single point.

We have presented the prime end theory which is the main ingredient in the proof of Theorem 1.2. The other ingredient is a carefully study of spirallike domains. To simplify the rest of the paper we introduce some notation. As usual, we make no distinction between a curve and its trace. Given $c \in \mathbb{C}$ with $\operatorname{Re} c>0$ and $w \in \mathbb{C}, w \neq 0$, we define the spiral $\operatorname{spir}_{c}[w]=\left\{e^{-c s} w: s \in \mathbb{R}\right\} \bigcup\{0\} \bigcup\{\infty\}$; given real numbers $s<t$, we define the spiral segment $\operatorname{spir}_{c}\left[e^{-s} w, e^{-t} w\right]$ as the subarc of $\operatorname{spir}_{c}[w]$ that goes from $e^{-s} w$ to $e^{-t} w$; finally, $\operatorname{spir}_{c}[w, 0]=\left\{e^{-c s} w: s \geq 0\right\} \bigcup\{0\}, \operatorname{spir}_{c}(w, 0]=$ $\left\{e^{-c s} w: s>0\right\} \bigcup\{0\}$, and $\operatorname{spir}_{c}[\infty, w]=\left\{e^{-c s} w: s \leq 0\right\} \bigcup\{\infty\}$.

Let us recall that given $c \in \mathbb{C}$ with $\operatorname{Re} c>0$, we say that $\Omega$ is $c$-spirallike (with respect to zero) if $0 \in \Omega$ and $e^{-c t} \Omega \subset \Omega$ for all $t \geq 0$, that is, the spiral $\operatorname{spir}_{c}[w, 0]$ is included in $\Omega$ for all $w \in \Omega$. When $c$ is a real number we have the so-called starlike domains.

In the rest of this section we show some properties of the boundary of a spirallike domain. The following easy-to-prove properties of spirallike domains will be used repeatedly in the paper:

1. If $e^{-s c} w, e^{-t c} w \in \partial_{\infty} \Omega$ with $s<t$, then $\operatorname{spir}_{c}\left[e^{-s} w, e^{-t} w\right] \subset \partial_{\infty} \Omega$.

2. If $w \in \partial_{\infty} \Omega \cap \mathbb{C}$, there is $u \in \partial_{\infty} \Omega \cap \mathbb{C}$ such that

$$
\operatorname{spir}_{c}[w] \bigcap \bar{\Omega}^{\mathbb{C}_{\infty}}=\operatorname{spir}_{c}(u, 0] \bigcup \operatorname{spir}_{c}\left[e^{c s} u, u\right],
$$

$$
\text { where } \operatorname{spir}_{c}(u, 0] \subset \Omega, \operatorname{spir}_{c}\left[e^{c s} u, u\right] \subset \partial_{\infty} \Omega \text {, and } s \in[0,+\infty] .
$$

Our next result gives information about the impression of a prime end in spirallike domains. 
Lemma 3.3 Let $p$ be a prime end of a c-spirallike domain $\Omega$. Then, the corresponding impression $I(p) \subset \partial_{\infty} \Omega$ must be of one of the following types:

1. $I(p)$ is a single point.

2. There is $\lambda \in \partial \mathbb{D}$ and $\eta_{1}<\eta_{2}$ such that $I(p)=\operatorname{spir}_{c}\left[e^{-\eta_{1} c} \lambda, e^{-\eta_{2} c} \lambda\right]$.

3. There is $\lambda \in \partial \mathbb{D}$ and $\eta \in \mathbb{R}$ such that $I(p)=\operatorname{spir}_{c}\left[\infty, e^{-\eta c} \lambda\right]$.

Proof. Suppose that diam ${ }^{\#}(I(p))>0$. Our first step is to show that there is $\lambda \in \mathbb{C}$ such that

$$
I(p) \subset \operatorname{spir}_{c}[\lambda]
$$

Take a zero-chain $\left(C_{m}\right) \in p$. For every $m \in \mathbb{N}$, define

$$
A_{m}:=\left(\bigcup\left\{\operatorname{spir}_{c}[w]: w \in \operatorname{ins} C_{m}\right\}\right) \bigcap \partial \mathbb{D} \text {. }
$$

Looking at the definition, it is plain that $A_{m+1} \subset A_{m}$, for $m \geq 1$.

We can assume that $\left\{C_{m}: m \in \mathbb{N}\right\}$ is a bounded subset of $\mathbb{C}$. Otherwise, there is a strictly increasing subsequence of natural numbers $\left(m_{j}\right)$ such that we can find $w_{j} \in C_{m_{j}}$ with $\left|w_{j}\right| \geq j$. Since $\lim _{m} \operatorname{diam}^{\#}\left(C_{m}\right)=0$, we see that $\lim _{j} \inf \left\{|w|: w \in C_{m_{j}}\right\}=+\infty$. This implies that $\lim _{j} \inf \{|z|: z \in$ ins $\left.\left(C_{m_{j}}\right)\right\}=+\infty$ and, therefore,

$$
I(p)=\bigcap_{j} \overline{\operatorname{ins}\left(C_{m_{j}}\right)} \mathbb{C}_{\infty}=\{\infty\} .
$$

This fact is in contradiction with the positivity of the spherical diameter of $I(p)$.

Since for bounded subsets in $\mathbb{C}$, the spherical metric is equivalent to the euclidean metric, we deduce that $\lim _{m} \operatorname{diam}\left(C_{m}\right)=0$. Now, given $w \in$ ins $C_{m}$ and, since $\operatorname{spir}_{c}[w, 0]$ is a connected subset of $\Omega$ which goes from ins $C_{m}$ into out $C_{m}$, we have that there is some non-zero $\widetilde{w} \in \operatorname{spir}_{c}[w, 0] \cap C_{m}$. Therefore, $\operatorname{spir}_{c}[w]=\operatorname{spir}_{c}[\widetilde{w}]$. Hence

$$
A_{m} \subset\left(\bigcup_{w \in C_{m}} \operatorname{spir}_{c}[w]\right) \bigcap \partial \mathbb{D} .
$$

From this, and using that $\inf \left\{|w|: w \in C_{m}, m \in \mathbb{N}\right\}>0$, we obtain that $\lim _{m} \operatorname{diam}\left(A_{m}\right)=0$.

So, we have built a decreasing sequence $\left(\overline{A_{m}}\right)$ of compact subsets of $\partial \mathbb{D}$ with diameters tending to zero. Necessarily, there is $\lambda \in \partial \mathbb{D}$ such that $\{\lambda\}=$ $\bigcap_{m} \overline{A_{m}}$ and, therefore, $I(p) \subset \operatorname{spir}_{c}[\lambda]$. Since the impression $I(p)$ is a continuum with positive diameter, we have that there are $\eta_{1}<\eta_{2}$ such that $I(p)=$ $\left\{\lambda e^{-s c}: s \in\left[\eta_{1}, \eta_{2}\right]\right\}$ or there is $\eta$ such that $I(p)=\left\{\lambda e^{-s c}: s \leq \eta\right\} \bigcup\{\infty\}$. 


\section{Proof of Theorem 1.2}

Proposition 4.1 Suppose $h: \mathbb{D} \rightarrow \mathbb{C}$ is a univalent function onto $\Omega$ and $\partial_{\infty} \Omega$ locally connected. If $f$ is a uniformly continuous map (in the spherical metric) of $\Omega$ into itself, then the map $g$ of $\mathbb{D}$ into $\mathbb{D}$ defined by $g=h^{-1} \circ f \circ h$ belongs to $A(\mathbb{D})$.

Proof. Let $a \in \partial \mathbb{D}$. We are going to prove that the function $g$ has a continuous extension to $a$. So, let

$$
U_{n}=\{z \in \mathbb{D}:|z-a|<1 / n\}
$$

for $n=1,2, \ldots$ Suppose that $\operatorname{diam}\left(g\left(U_{n}\right)\right)$ does not converge to 0. Since $g\left(U_{n}\right)$ is connected, we can find Jordan $\operatorname{arcs} C_{n}$ in $g\left(U_{n}\right)$ and $\varepsilon>0$ such that $\operatorname{diam}\left(C_{n}\right) \geq \varepsilon$ for all $n$.

On the other hand, the continuity of $h$ from $\overline{\mathbb{D}}$ into $\mathbb{C}_{\infty}$ implies that

$$
\lim _{n} \operatorname{diam}^{\#}\left(h\left(U_{n}\right)\right)=0 .
$$

Moreover, the uniform continuity of $f$ implies that

$$
\lim _{n} \operatorname{diam}^{\#}\left(f\left(h\left(U_{n}\right)\right)\right)=0
$$

and thus there is a point $c \in \bar{\Omega}^{\mathbb{C}_{\infty}}$ such that

$$
\lim _{n \rightarrow \infty} \sup \left\{|w-c|: w \in f\left(h\left(U_{n}\right)\right)\right\}=0 .
$$

Since $h \circ g=f \circ h$, we have that $f\left(h\left(U_{n}\right)\right)=h\left(g\left(U_{n}\right)\right)$ and

$$
\lim _{n \rightarrow \infty} \sup \left\{|w-c|: w \in h\left(g\left(U_{n}\right)\right)\right\}=0 .
$$

In particular,

$$
\lim _{n \rightarrow \infty} \sup \left\{|w-c|: w \in h\left(C_{n}\right)\right\}=0 .
$$

Summing up, we have a sequence of Jordan $\operatorname{arcs} C_{n}$ in $\mathbb{D}, \varepsilon>0$ such that $\operatorname{diam}\left(C_{n}\right) \geq \varepsilon$ for all $n$ and $h\left(C_{n}\right)$ tends to a point $c \in \bar{\Omega}^{\mathbb{C}_{\infty}}$. That is, $\left(C_{n}\right)$ is a sequence of Koebe arcs with respect to $h$. This is a contradiction because a univalent function is normal [7, Lemma 9.3] and by a theorem of Bagemihl and Seidel a non-constant normal function has no sequence of Koebe arcs [7, Corollary 9.1].

Therefore we have that $\lim _{n} \operatorname{diam} g\left(U_{n}\right)=0$, which implies that $g$ has a continuous extension to $a$. Since $a \in \partial \mathbb{D}$ is arbitrary, we have that $g \in A(\mathbb{D})$. 
Proof of Theorem 1.2. Without lose of generality, we assume that the DW-point of $\Phi$ is $b=0$.

It is clear that (1) implies (3).

$(2) \Longrightarrow(1)$. According to Theorem 1.1, we have to show that $\varphi_{t} \in A(\mathbb{D})$, for all $t \geq 0$, and $\varphi_{t}(z) \rightarrow z$ as $t$ tends to $0^{+}$, for all $z \in \partial \mathbb{D}$. Since $\partial_{\infty} \Omega$ is a locally connected subset of $\mathbb{C}_{\infty}$, we can assume that $h$ has a continuous extension (which we still denote by $h$ ) from $\overline{\mathbb{D}}$ onto $\bar{\Omega}^{\mathbb{C}_{\infty}}$. Therefore, the impression of the prime end $\widehat{h}(z)$ is exactly $h(z)$. We recall that $\widehat{h}$ is the associated function to $h$ in the prime end theorem.

To get that $\varphi_{t} \in A(\mathbb{D})$, for all $t \geq 0$, we just have to apply Proposition 4.1 noticing that the function $w \mapsto e^{-c t} w$ is continuous in the Riemann sphere and so it is a uniformly continuous map (in the spherical metric) of $\Omega$ into itself.

Once we know that each $\varphi_{t} \in A(\mathbb{D})$, we are going to show that $\varphi_{t}(z) \rightarrow z$ as $t$ tends to 0 , for all $z \in \partial \mathbb{D}$. We fix $z \in \partial \mathbb{D}$ and distinguish three cases:

Case I: $h(z)=\infty$.

In this case, we are going to see that

$$
\lim _{\xi \rightarrow z, \xi \in \mathbb{D}} \varphi_{t}(\xi)=z .
$$

In particular, $z$ is a boundary fixed point for $\varphi_{t}$, that is, $\varphi_{t}(z)=z$, for all $t \geq 0$, and obviously, $\varphi_{t}(z) \rightarrow z$ as $t$ tends to 0 .

Take a sequence $z_{n} \rightarrow z$, with $z_{n} \in \mathbb{D}$. Take also a zero-chain $\left(C_{m}\right)$ in $\mathbb{D}$ converging to $z$ such that $\left(h\left(C_{m}\right)\right)$ is a zero-chain in $\Omega$ with the end points of each crosscut $h\left(C_{m}\right)$ different from $\infty$. Clearly, each ${\overline{h\left(C_{m}\right)}}^{\mathbb{C}_{\infty}}=\overline{h\left(C_{m}\right)}$ is a compact subset of $\mathbb{C}$ and this allows us to define the following two real numbers:

$$
\begin{aligned}
& \beta_{m}:=\max \left\{|w|: w \in \overline{h\left(C_{m}\right)}\right\} \in(0,+\infty) \\
& \gamma_{m}:=\min \left\{|w|: w \in \overline{h\left(C_{m}\right)}\right\} \in(0,+\infty) .
\end{aligned}
$$

For every $m \in \mathbb{N}$, choose $w_{m} \in \overline{h\left(C_{m}\right)}$ with $\left|w_{m}\right|=\gamma_{m}$. Let us see that $\lim _{m} \gamma_{m}=+\infty$. Otherwise, we can find $M>0$ and a subsequence $\left(\gamma_{m_{k}}\right)_{k}$ with $\gamma_{m_{k}} \leq M$, for all $k \in \mathbb{N}$. Hence, $\inf \left\{\mathrm{d}^{\#}\left(\infty, w_{m_{k}}\right): k \in \mathbb{N}\right\}>0$. Since $h(z)=\infty$, we have that $\infty \in \bigcap_{m} \overline{\operatorname{ins}\left(h\left(C_{m}\right)\right)} \mathbb{C}_{\infty}$. Recalling that $\partial_{\infty} \Omega$ is a locally connected subset of $\mathbb{C}_{\infty}$, we get a contradiction because

$$
\begin{aligned}
0<\inf \left\{\mathrm{d}^{\#}\left(\infty, w_{m_{k+1}}\right): k \in \mathbb{N}\right\} & \leq \lim _{k} \mathrm{~d}^{\#}\left(\infty, w_{m_{k+1}}\right) \\
& \leq \lim _{k} \operatorname{diam}^{\#}\left(\overline{\operatorname{ins}\left(h\left(C_{m_{k}}\right)\right)} \mathbb{C}^{\mathbb{C}_{\infty}}\right)=0 .
\end{aligned}
$$


Therefore, for each $m \in \mathbb{N}$, we can find $k_{m}>m$ such that $e^{-t \operatorname{Rec}} \gamma_{k_{m}}>\beta_{m}$. We are going to check that $e^{-t c}$ ins $h\left(C_{k_{m}}\right) \subset \operatorname{ins} h\left(C_{m}\right)$. Take $w \in \operatorname{ins} h\left(C_{k_{m}}\right)$. Since $\Omega$ is $c$-spirallike, the definition of the numbers $\gamma_{m}$ implies that $|w| \geq \gamma_{k_{m}}$.

Moreover, by the choice of $k_{m}$, the spiral $\operatorname{spir}_{c}\left[w, e^{-t c} w\right]$ does not cut the crosscut $h\left(C_{m}\right)$. Otherwise, there exists $e^{-s c} w \in h\left(C_{m}\right)$ with $0 \leq s \leq t$. Then

$$
\beta_{m} \geq\left|e^{-s c} w\right|=e^{-s \operatorname{Rec}}|w| \geq e^{-s \operatorname{Re} c} \gamma_{k_{m}} \geq e^{-t \operatorname{Rec}} \gamma_{k_{m}}>\beta_{m} .
$$

What is more, the spiral $\operatorname{spir}_{c}\left[w, e^{-t c} w\right]$ is a connected subset contained in $\Omega$ and $w \in \operatorname{ins} h\left(C_{m}\right)\left(k_{m}>m\right)$, thus $\operatorname{spir}_{c}\left[w, e^{-t c} w\right] \subset \operatorname{ins} h\left(C_{m}\right)$. In particular, $e^{-t c} w \in \operatorname{ins} h\left(C_{m}\right)$. That is, $e^{-t c}$ ins $h\left(C_{k_{m}}\right) \subset$ ins $h\left(C_{m}\right)$.

Let us fix a natural number $m \in \mathbb{N}$. Since $\left(z_{n}\right)$ is convergent to $z$, we can obtain $n_{0} \in \mathbb{N}$ such that if $n \geq n_{0}$, we have $z_{n} \in \operatorname{ins} C_{k_{m}}$. Then

$$
e^{-t c} h\left(z_{n}\right) \in e^{-t c} \operatorname{ins} h\left(C_{k_{m}}\right) \subset \operatorname{ins} h\left(C_{m}\right)=h\left(\operatorname{ins} C_{m}\right) .
$$

So $\varphi_{t}\left(z_{n}\right) \in \operatorname{ins} C_{m}$. Since $\left(C_{m}\right)$ is a zero-chain in $\mathbb{D}$ converging to $z$, we deduce finally that $\lim _{n} \varphi_{t}\left(z_{n}\right)=z$.

Case II: $h(z) \in \mathbb{C}$ and has exactly one pre-image in $\partial \mathbb{D}$.

Take a sequence $t_{n} \rightarrow 0$, with $t_{n}>0$.. Since $\varphi_{t_{n}} \in A(\mathbb{D})$, we can find $r_{n} \in(1-1 / n, 1)$ such that $\left|\varphi_{t_{n}}\left(r_{n} z\right)-\varphi_{t_{n}}(z)\right| \leq 1 / n$. Using Lemma 3.1, we have that $h^{-1}$ has a continuous extension from $\Omega \bigcup\{h(z)\}$ into $\overline{\mathbb{D}}$. Therefore,

$$
\varphi_{t_{n}}\left(r_{n} z\right)=h^{-1}\left(e^{-t_{n} c} h\left(r_{n} z\right)\right) \stackrel{n \rightarrow+\infty}{\longrightarrow} h^{-1}(h(z))=z .
$$

Finally,

$$
\left|\varphi_{t_{n}}(z)-z\right| \leq \frac{1}{n}+\left|\varphi_{t_{n}}\left(r_{n} z\right)-z\right| \stackrel{n \rightarrow+\infty}{\longrightarrow} 0
$$

Case III: $h(z) \in \mathbb{C}$ and has more than one pre-image in $\partial \mathbb{D}$.

We take a zero-chain $\left(C_{m}\right)$ in $\mathbb{D}$ converging to $z$ such that $\left(h\left(C_{m}\right)\right)$ is a zero-chain in $\Omega$ with the end points of each crosscut $h\left(C_{m}\right)$ different from the point $h(z)$. For each $m \in \mathbb{N}$, we define $\delta_{m}:=\operatorname{dist}\left(h(z), h\left(C_{m}\right)\right)$ which is a positive number by the election of $h\left(C_{m}\right)$.

Moreover, by the continuity of $h$ in $\overline{\mathbb{D}}$, we have that $\delta_{m} \stackrel{m \rightarrow+\infty}{\longrightarrow} 0$. In addition, recalling that the zero-chain $\left(C_{m}\right)$ converges to $z$ and by the continuity of $h$ in $\overline{\mathbb{D}}$, we take for each $m$, a number $s_{m} \in(0,1)$ such that

$$
|h(r z)-h(z)|<\frac{\delta_{m}}{2} \text { and } h(r z) \in \operatorname{ins} h\left(C_{m}\right), \text { for all } r \in\left(s_{m}, 1\right) .
$$

We also take for each $m$, a number $\rho_{m}>0$ such that

$$
|h(z)|\left|e^{-t c}-1\right|<\frac{\delta_{m}}{2}, \text { for all } t \in\left[0, \rho_{m}\right]
$$


Then, if $r \in\left(s_{m}, 1\right)$ and $t \in\left[0, \rho_{m}\right]$, we obtain that

$$
\begin{aligned}
\left|e^{-t c} h(r z)-h(z)\right| & \leq\left|e^{-t c} h(r z)-e^{-t c} h(z)\right|+\left|e^{-t c} h(z)-h(z)\right| \\
& \leq\left|e^{-t c}\right||h(r z)-h(z)|+|h(z)|\left|e^{-t c}-1\right|<\delta_{m} .
\end{aligned}
$$

And, therefore,

$$
\operatorname{spir}_{c}\left[h(r z), e^{-\rho_{m} c} h(r z)\right] \subset B\left(h(z), \delta_{m}\right) \bigcap \Omega .
$$

Bearing in mind how $\delta_{m}$ was chosen, this means that $\operatorname{spir}_{c}\left[h(r z), e^{-\rho_{m} c} h(r z)\right]$ can not cut $h\left(C_{m}\right)$. Since $\operatorname{spir}_{c}\left[h(r z), e^{-\rho_{m} c} h(r z)\right]$ is a connected subset of $\Omega$ and $h(r z) \in \operatorname{ins} h\left(C_{m}\right)$, we obtain that

$$
\operatorname{spir}_{c}\left[h(r z), e^{-\rho_{m} c} h(r z)\right] \subset \operatorname{ins} h\left(C_{m}\right) .
$$

Take two arbitrary sequences $t_{n} \rightarrow 0$, with $t_{n}>0$ and $r_{n} \rightarrow 1$, with $r_{n} \in(0,1)$. Fix $m \in \mathbb{N}$ and let $s_{m}$ and $\rho_{m}$ be as above. Obviously, we can find $n_{0}$ such that for $n \geq n_{0}, r_{n} \in\left(s_{m}, 1\right)$ and $t_{n} \in\left(0, \rho_{m}\right)$. Then

$$
\varphi_{t_{n}}\left(r_{n} z\right) \in h^{-1}\left(\operatorname{spir}_{c}\left[h(r z), e^{-\rho_{m} c} h(r z)\right]\right) \in h^{-1}\left(\operatorname{ins} h\left(C_{m}\right)\right)=\operatorname{ins} C_{m} .
$$

Since $\left(C_{m}\right)$ is a zero-chain converging to $z$, we have obtained that $\varphi_{t_{n}}\left(r_{n} z\right) \rightarrow z$ as $n$ tends to infinite. Since $\varphi_{t_{n}} \in A(\mathbb{D})$, we conclude that $\lim _{n} \varphi_{t_{n}}(z)=z$, following a similar argument to the one given in Case II.

$(2) \Longrightarrow(3)$. We have to prove that if $\partial_{\infty} \Omega$ is not locally connected, then there is $t>0$ such that $\varphi_{t} \notin A(\mathbb{D})$ or, equivalently, such that $\varphi_{t}$ has no continuous extension to $\mathbb{D} \bigcup\{z\}$, for some $z \in \partial \mathbb{D}$.

If $\partial_{\infty} \Omega$ is not locally connected, the continuity theorem tell us that $h$ does not admit a continuous extension from $\overline{\mathbb{D}}$ into $\bar{\Omega}^{\mathbb{C}_{\infty}}$. So, there exists $z \in \partial \mathbb{D}$ such that $h$ does not admit a continuous extension from $\mathbb{D} \bigcup\{z\}$ into $\bar{\Omega}^{\mathbb{C}_{\infty}}$. Therefore, the impression of the prime end $\widehat{h}(z)$ can not be singleton. If $I(\widehat{h}(z))$ is not singleton, necessarily $\operatorname{diam}^{\#} I(\widehat{h}(z))>0$ and, by Lemma 3.3, we have that there are $\lambda \in \partial \mathbb{D}$ and $\eta_{1}<\eta_{2}\left(\eta_{1}, \eta_{2} \in \mathbb{R}\right)$ such that $I(\widehat{h}(z))=\left\{\lambda e^{-s c}: s \in\left[\eta_{1}, \eta_{2}\right]\right\}$ or there are $\lambda \in \partial \mathbb{D}$ and $\eta_{2} \in \mathbb{R}$ such that $I(\widehat{h}(z))=\left\{\lambda e^{-s c}: s \leq \eta_{2}\right\} \bigcup\{\infty\}$.

We take a zero-chain $\left(C_{m}\right)$ in $\mathbb{D}$ converging to $z$ such that $\left(h\left(C_{m}\right)\right)$ is a zero-chain in $\Omega$. Therefore, $\left(h\left(C_{m}\right)\right) \in \widehat{h}(z)$. Intuitively, $\lambda e^{-\eta_{2} c}$ must be a limit of end points of the crosscuts $h\left(C_{m}\right)$. More precisely, let us show that:

$(R) \quad$ If $w_{m} \in h\left(C_{m}\right)(m \in \mathbb{N})$, then $\lim _{m} w_{m}$ always exists and it is equal to $\lambda e^{-\eta_{2} c}$. 
Take $w_{m}=h\left(\xi_{m}\right)$ with $\xi_{m} \in C_{m}$. Clearly $\xi_{m} \rightarrow z$. In order to prove $(R)$, it is enough to check that $\left(w_{m}\right)$ has a subsequence converging to $\lambda e^{-\eta_{2} c}$. We may assume that $\left\{h\left(C_{m}\right): m \in \mathbb{N}\right\}$ is a bounded subset of $\mathbb{C}$ (see the proof of Lemma 3.3), so we have a subsequence (that we still denote $\left(\xi_{m}\right)$ ) such that $\lim _{m} h\left(\xi_{m}\right) \in I(\widehat{h}(z)) \backslash\{\infty\}$. Hence, $\lim _{m} w_{m}=\lambda e^{-s c}$, with $s \leq \eta_{2}$. Suppose that $s<\eta_{2}$ and define

$$
\delta:=\min \left\{\left|e^{-\eta_{2} c}-e^{-s c}\right|, \frac{\operatorname{Re} c}{|c|} \ell\left(s, \eta_{2}\right)\right\}>0 .
$$

We have used the notation

$$
\ell\left(r_{0}, r_{1}\right)=\int_{r_{0}}^{r_{1}}\left|c e^{-t c}\right| d t=\frac{|c|}{\operatorname{Rec}}\left(e^{-r_{0} \operatorname{Rec}}-e^{-r_{1} \operatorname{Re} c}\right), r_{0} \leq r_{1},
$$

for the length of an arbitrary spiral $\operatorname{spir}_{c}\left[e^{-r_{0} c} \lambda, e^{-r_{1} c} \lambda\right]$.

Since $\lim _{m} \operatorname{diam}\left(h\left(C_{m}\right)\right)=0$, there is $m_{0} \in \mathbb{N}$ such that, $\operatorname{diam}\left(h\left(C_{m}\right)\right) \leq \frac{\delta}{4}$, for all $m \geq m_{0}$. On the other hand, take $m_{1} \geq m_{0}$ such that, if $m \geq m_{1}$, then $\left|w_{m}-\lambda e^{-s c}\right| \leq \frac{\delta}{4}$. In particular,

$$
\left\{h\left(C_{m}\right): m \geq m_{1}\right\} \subset B\left(\lambda e^{-s c}, \frac{\delta}{2}\right) .
$$

Since $\lambda e^{-\eta_{2} c} \in I(\widehat{h}(z))$, we can find $\alpha_{m} \in \operatorname{ins} C_{m}$ with $\alpha_{m} \rightarrow z$ and $h\left(\alpha_{m}\right) \rightarrow \lambda e^{-\eta_{2} c}$. For each $m \in \mathbb{N}$, the $\operatorname{spiral}_{\operatorname{spir}_{c}}\left[h\left(\alpha_{m}\right), 0\right]$ goes from ins $h\left(C_{m}\right)$ to out $h\left(C_{m}\right)$, so it cuts $h\left(C_{m}\right)$ in some $e^{-r_{m} c} h\left(\alpha_{m}\right)$, where $r_{m}>0$. Passing to a subsequence (without changing the notation), we have that $r_{m} \rightarrow r \in[0,+\infty]$. Bearing in mind $(*)$, we see that $r \neq+\infty$ and

$$
e^{-r c} \lambda e^{-\eta_{2} c} \in \overline{B\left(\lambda e^{-s c}, \frac{\delta}{2}\right)} \text {. }
$$

Hence,

$$
\frac{\operatorname{Re} c}{|c|} \ell\left(s, \eta_{2}+r\right)=\left|e^{-\left(\eta_{2}+r\right) \operatorname{Rec}}-e^{-s \operatorname{Re} c}\right| \leq\left|e^{-\left(\eta_{2}+r\right) c}-e^{-s c}\right| \leq \frac{\delta}{2}<\frac{\operatorname{Re} c}{2|c|} \ell\left(s, \eta_{2}\right) .
$$

Since $r \geq 0$, we deduce that $\ell\left(s, \eta_{2}\right) \leq \ell\left(s, \eta_{2}+r\right) \leq(1 / 2) \ell\left(s, \eta_{2}\right)$, and we arrive to a contradiction because $\ell\left(s, \eta_{2}\right)>0$. Thus, $(R)$ is satisfied.

We continue the proof only for the "[$\left.\eta_{1}, \eta_{2}\right]$-case". The " $\eta_{2}$-case" can be proved with similar arguments. Take $0<t<\eta_{2}-\eta_{1}$ and, assume that $\varphi_{t}$ has a continuous extension to $\mathbb{D} \bigcup\{z\}$.

Since $\lambda e^{-c \eta_{1}}, \lambda e^{-c \eta_{2}} \in I(\widehat{h}(z))$ and recalling that $\left(C_{m}\right)$ is a zero-chain convergent to $z$, we can find $a_{m}, b_{m} \in \operatorname{ins} C_{m}$, such that $a_{m} \rightarrow z, h\left(a_{m}\right) \rightarrow$ $\lambda e^{-\eta_{2} c}, b_{m} \rightarrow z$, and $h\left(b_{m}\right) \rightarrow \lambda e^{-\eta_{1} c}$. Obviously, $e^{-t c} h\left(b_{m}\right) \rightarrow \lambda e^{-\left(\eta_{1}+t\right) c}$. 
Let us see that there is $m_{0} \in \mathbb{N}$ such that $e^{-t c} h\left(b_{m}\right) \in \operatorname{ins} h\left(C_{m}\right)$, for every $m \geq m_{0}$. If this were not true and since $h\left(b_{m}\right) \in \operatorname{ins} h\left(C_{m}\right)$, there would be a strictly increasing subsequence $\left(m_{j}\right)$ such that

$$
e^{-t c} h\left(b_{m_{j}}\right) \in \operatorname{out} h\left(C_{m_{j}}\right) \bigcup h\left(C_{m_{j}}\right)
$$

Moreover, the spiral $\operatorname{spir}_{c}\left[h\left(b_{m_{j}}\right), e^{-t c} h\left(b_{m_{j}}\right)\right]$ cuts $h\left(C_{m_{j}}\right)$ in some point $e^{-s_{j} c} h\left(b_{m_{j}}\right)$, with $s_{j} \in(0, t]$. Passing to a subsequence (keeping the notation), we have that $s_{j} \rightarrow s \in[0, t]$. Therefore, using $(R)$, we obtain that

$$
e^{-s c} \lambda e^{-\eta_{1} c}=\lim _{j} e^{-s_{j} c} h\left(b_{m_{j}}\right)=e^{-\eta_{2} c} \lambda .
$$

Clearly, we obtain a contradiction because $s \in[0, t]$ and $t \in\left(0, \eta_{2}-\eta_{1}\right)$.

Since $\left(h\left(C_{m}\right)\right)_{m \geq m_{0}} \in \widehat{h}(z)$, we have shown that $e^{-c t} h\left(b_{m}\right) \rightarrow \widehat{h}(z)$ in Carathéodory's topology of $\widehat{\Omega}$. Hence

$$
\lim _{m} \varphi_{t}\left(b_{m}\right)=\lim _{m} h^{-1}\left(e^{-t c} h\left(b_{m}\right)\right)=(\widehat{h})^{-1}(\widehat{h}(z))=z .
$$

Since we are assuming that $\varphi_{t}$ has a continuous extension to $\mathbb{D} \bigcup\{z\}$, we get $\lim _{n} \varphi_{t}\left(a_{m}\right)=z$. On the other hand, if $\varphi_{t}\left(a_{m}\right) \rightarrow z$, the limit of $h\left(\varphi_{t}\left(a_{m}\right)\right)$ must be in the impression of $\widehat{h}(z)$. But, this is absurd, because

$$
h\left(\varphi_{t}\left(a_{m}\right)\right)=e^{-t c} h\left(a_{m}\right) \rightarrow \lambda e^{-c\left(\eta_{2}+t\right)} \notin I(\widehat{h}(z)) .
$$

\section{References}

[1] Berkson, E. and Porta, H.: Semigroups of analytic functions and composition operators. Michigan Math. J. 25 (1978), 101-115.

[2] Conway, J. B.: Functions of one complex variable. Second edition. Graduate Texts in Mathematics 11. Springer-Verlag, New York, 1978.

[3] Conway, J. B.: Functions of one complex variable II. Graduate Texts in Mathematics 159. Springer-Verlag, New York, 1995.

[4] Diestel, J.: Sequences and Series in Banach Spaces. Graduate Texts in Mathematics 92. Springer-Verlag, New York, 1984.

[5] Dunford, N. And Schwartz, J. T.: Linear Operators. Part I: General Theory. Interscience Publishers, New York, 1958.

[6] Milnor, J.: Dynamics in one complex variable. Vieweg Verlag, Weisbaden, 2000.

[7] Pommerenke, Ch.: Univalent functions. Studia Mathematica 25. Vandenhoeck \& Ruprecht, Göttingen, 1975. 
[8] Pommerenke, Ch.: Boundary behaviour of conformal maps. Fundamental Principles of Mathematical Sciences 299. Springer-Verlag, Berlin, 1992.

[9] Shapiro, J. H.: Composition operators and classical function Theory. Universitext: Tracts in Mathematics. Springer-Verlag, New York, 1993.

[10] Shoikhet, D.: Semigroups in geometrical function theory. Kluwer Academic Publishers, Dordrecht, 2001.

[11] Siskakis, A. G.: Semigroups of composition operators on spaces of analytic functions, a review. In Studies on composition operators (Laramie, 1996), 229-252. Contemp. Math. 213. Amer. Math. Soc., Providence, RI, 1998.

[12] Yosida, K.: Functional Analysis. Sixth Edition. Fundamental Principles of Mathematical Sciences 123. Springer-Verlag, Berlin-New York, 1980.

[13] ZHU, K.: Operator Theory in Function spaces. Monographs and Textbooks in Pure and Applied Mathematics 139. Marcel Dekker, New York, 1990.

Recibido: 24 de julio de 2003

Revisado: 26 de febrero de 2004

Manuel D. Contreras

Camino de los Descubrimientos, s/n Departamento de Matemática Aplicada II

Escuela Superior de Ingenieros

Universidad de Sevilla Sevilla, 41092, Spain.

contreras@esi.us.es

Santiago Díaz-Madrigal

Camino de los Descubrimientos, s/n

Departamento de Matemática Aplicada II

Escuela Superior de Ingenieros

Universidad de Sevilla

Sevilla, 41092, Spain.

madrigal@us.es

This research has been partially supported by the Ministerio de Ciencia y Tecnología and the European Union (FEDER) projects n. BFM2000-1062 and BFM2003-07294-C02-02 and by La Consejería de Educación y Ciencia de la Junta de Andalucía. 